\title{
Microparticles and vascular dysfunction in obstructive sleep apnoea
}

\author{
Wojciech Trzepizur ${ }^{1,2}$, Maria Carmen Martinez ${ }^{1}$, Pascaline Priou ${ }^{1,2}$, \\ Ramaroson Andriantsitohaina ${ }^{1}$ and Frédéric Gagnadoux ${ }^{1,2}$ \\ Affiliations: \\ ${ }^{1}$ INSERM U1063, Angers, and \\ ${ }^{2}$ Dept of Respiratory Diseases, Angers University Hospital, Angers, France.
}

Correspondence:

Wojciech Trzepizur, Université d’Angers, CHU Angers, Département de Pneumologie, 4 rue Larrey, 49033 Angers Cedex, France. E-mail: wotrzepizurdchu-angers.fr

ABSTRACT Obstructive sleep apnoea (OSA) is independently associated with various cardiovascular diseases, including myocardial infarction and stroke. OSA may promote atherosclerosis risk factors such as hypertension, diabetes and dyslipidaemia, and may have direct proatherogenic effects on the vascular wall. A growing number of studies have recently focused on the role of microparticles (MPs) in the atherogenic process. MPs are small plasma membrane vesicles that can be released by a variety of vascular or blood cells, and contain both membrane and cytosolic elements. Case-control studies have shown that platelet-, endothelium- and leukocyte-derived MP levels are increased in OSA. Experimental evidence has demonstrated that MPs from OSA patients induce endothelial dysfunction, inflammation and vascular hyperreactivity when injected into mice. In this review, we provide an overview of the main characteristics of MPs, their expression in OSA and their potential role in the atherogenic process associated with OSA.

@ERSpublications

Overview of microparticles, and their expression in OSA and potential role in the atherogenic process in OSA http://ow.ly/toiAV 


\section{Introduction}

Obstructive sleep apnoea (OSA) is a highly prevalent disease characterised by recurrent episodes of partial or complete obstruction of the upper airways during sleep, leading to repeated falls in oxygen saturation, increased negative intrathoracic pressure and frequent arousals from sleep. There is clear evidence for an independent association between OSA and cardiovascular events [1-3]. A higher rate of incident coronary heart diseases [4] and strokes [5] was demonstrated in male patients with severe OSA. Recent data show that OSA may also contribute to cardiac systolic [6] and diastolic dysfunction [7], as well as a higher rate of cardiac arrhythmias [8]. Data from clinical and experimental studies suggest that intermittent hypoxia is the main component linking OSA to atherosclerosis $[3,9,10]$. Intermittent hypoxia induces endothelial dysfunction, systemic vascular inflammation, oxidative stress and vascular smooth cell activation, and promotes various vascular risk factors such as dyslipidaemia [11, 12], insulin resistance [13] and hypertension [14].

Activated or apoptotic cells release different types of membrane vesicles, including microparticles (MPs), exosomes and apoptotic bodies. Exosomes $(<100 \mathrm{~nm}$ in diameter) are produced by multivesicular bodies during endocytosis. Apoptotic bodies might be generated during the final steps of programmed cell death. These different vesicles are distinguished from one another on the basis of their subcellular origin, their size, their content, the mechanisms leading to their formation and, from a practical point of view, how they are isolated. This review will focus on the role of MPs in the vascular dysfunction in OSA.

MPs are defined by their size, which ranges between 0.1 and $1 \mu \mathrm{m}$, and their distinctive lipid layer composition, which is rich in negatively charged phospholipids, particularly phosphatidylserine. Their composition depends on both their cellular origin and the stimuli involved during their generation. MPs may contain membrane and cytosolic proteins, transcription factors, and genetic material such as ribosomal RNA, messenger RNA and microRNA, as well as lipids or organelles from their cells of origin [15, 16]. MPs can convey biological messages to target cells through a range of pathways. Ligands carried by MPs can directly interact with receptors on target cells and induce signal transduction. In addition, membranes of MPs can fuse with the plasma membrane of target cells, leading to transfer of membrane components and delivery of MP cytoplasmic content. Finally, MPs may be phagocytosed and internalised into recipient cells [17]. Any of these interactions can result in activation or inhibition of intracellular pathways in target cells or modification of their phenotype [18].

Several recent reviews have focused on the critical role of MPs as biomarkers and communication shuttles between cells [19-21].

MPs are present under normal physiological conditions and are involved in tightly controlled biological functions, including inflammation [22], haemostasis [23] and angiogenesis [21]. The protective or deleterious effects of MPs in different clinical situations are still discussed. For example, MPs are released during cardiac stress (dobutamine echocardiography) and this rise is diminished in patients with vascular disease compared with healthy subjects, suggesting that MP increase may represent an adaptive response to a specific stimulus rather than a pathological process [24]. MPs isolated from patients with septic shock have been shown to exert a protective role in vascular function $[25,26]$.

However, MPs are more usually considered independent functional pathological effectors in a large variety of diseases, including cardiovascular and metabolic disorders such as coronary heart disease [27, 28], hypertension [29] and diabetes [30], and severe pulmonary diseases such as acute lung injury [31] and pulmonary hypertension [32]. Furthermore, MPs that harbour markers of cellular activation have been shown to predict poor cardiovascular outcomes in at-risk patients [33, 34].

This article reviews clinical studies of MPs in OSA patients and experimental data supporting the potential contribution of MPs in OSA-associated vascular dysfunction.

\section{Technical aspects of characterising circulating MPs}

Characterising MPs is technically challenging because of their small size, heterogeneous densities, and overlap with other particulate structures and platelets. MPs carry antigenic markers characteristic of their parent cell, which is exploited for identifying their cellular origin, usually by fluorescently labelled monoclonal antibodies using flow cytometry or, more rarely, an ELISA.

MP levels may rise and decrease quickly after specific stimuli. A single high-fat meal is sufficient to elicit a pulse of MPs [35], as is application of a blood pressure cuff, exercise [36] or a cardiac stress (dobutamine echocardiography) [24]. Then, clearance from the circulation can occur during the next hour [24]. Therefore, the time of venepuncture is of particular interest and a morning fasting blood sample is usually preferred to facilitate further comparison between studies. 
A serious obstacle in the comparison of results from different clinical studies is the variety of methods used to recover MPs. Differences may occur at each step of the MP collection process: use of anticoagulants after venepuncture, storage conditions, length and number of freeze-thaw cycles, centrifugation, washing, and pelleting [37]. The use of these different methods accounts for the frequently divergent reports found in the literature. Several considerations are of special importance.

First, there are wide differences in pre-analytical variables, including the anticoagulant used for blood collection, the time before the first centrifugation, agitation during tube transportation and the centrifuge speeds used to eliminate whole cells or to sediment the MPs [37, 38]. Laboratories that use higher centrifuge speeds to eliminate whole cells may discard materials that are measured as MPs in other laboratories. Indeed, numerous data in the literature identify centrifugation as the main factor affecting MP analysis [39-41]. Regarding these large differences in pre-analytical protocols, the International Society on Thrombosis and Haemostasis has recently proposed recommendations for the standardisation of preanalytical steps [42]. They recommend the use of citrated tubes, that processing should occur $<2 \mathrm{~h}$ after blood collection, the use of two centrifugations at $2500 \times \mathrm{g}$ for $15 \mathrm{~min}$ and storage at $-80^{\circ} \mathrm{C}$.

The second consideration is the large variety of antigenic markers employed for counting and identifying the MP cell origin. MPs from the same cell type can display multiple antigenic phenotypes, which can have differential antigen enrichment and may reflect different clinical states. For example, the counts of endothelium-derived microparticles (EMPs) can differ depending not only in the labelled markers used but also in how the cells were stimulated to release the EMPs [43]. Specifically, EMPs from cultured endothelial cells showed clear differences in their membrane markers depending on whether the cells were apoptotic or activated by TNF- $\alpha$ [43]. These in vitro findings were subsequently reflected in patient studies. In coronary artery disease, EMPs released during the acute phases of ischaemia differed from those in the chronic, stable phases. $\mathrm{CD} 1^{+}$EMP levels were markedly elevated in patients with acute coronary syndrome but not in patients with stable angina. In contrast, $\mathrm{CD} 51^{+}$EMP levels were increased in patients with both acute coronary syndrome and stable angina [44].

There is a mistaken view held by some investigators that MPs positive for annexin V (AV) reflect the total count of MPs, considering that all MPs contain phosphatidylserine. Recent data suggest that the fraction of MPs that bind AV varies widely, from a few percent for MPs from activated cells to $>80 \%$ for MPs from apoptotic cells [45].

Another analytical variable comes from flow cytometry. Fluorescent antibody aggregates, present in commercial antibody solutions, may have the same scatter properties as MPs and contribute to the identification of false MPs during flow cytometry analysis [46, 47]. In a recent review, LACROIX et al. [48] suggested that centrifugation of antibodies before use may reduce this interference.

\section{Clinical studies of MPs in OSA}

Case-control studies investigating MPs in OSA are summarised in table 1 for pre-analytical procedures and table 2 for MP levels and phenotypes. All the venepunctures were performed in the morning after sleep recording in fasting patients. Some studies proposed additional venepunctures at 04:00 $\mathrm{h}$ [49] and 17:00 $\mathrm{h}$ [56] to access the night to morning or the evening to morning changes. Two studies used EDTA tubes

TABLE 1 Pre-analytical procedures involved in clinical studies of circulating microparticles (MPs) in obstructive sleep apnoea

\begin{tabular}{|c|c|c|c|c|c|c|}
\hline $\begin{array}{l}\text { First author } \\
\text { [ref.] }\end{array}$ & Year & Anticoagulant & Preparation of PPP & $\begin{array}{c}\text { Sedimentation of } \\
\text { MPs }\end{array}$ & Storage & Wash step \\
\hline GEISER [49] & 2002 & Citrate & No & No & Formaldehyde & No \\
\hline AYERS [51] & 2009 & Citrate & $1550 \times g, 20 \mathrm{~min}$ & $18000 \times g, 30 \mathrm{~min}$ & $-80^{\circ} \mathrm{C}$ & Yes \\
\hline PRIOU [52] & 2010 & EDTA & $\begin{array}{l}270 \times g, 20 \mathrm{~min} \\
1500 \times \mathrm{g}, 20 \mathrm{~min}\end{array}$ & No & $-80^{\circ} \mathrm{C}$ & No \\
\hline КІм [54] & 2011 & & $\begin{array}{l}2000 \times \mathrm{g}, 20 \mathrm{~min} \\
20000 \times \mathrm{g}, 90 \mathrm{~min}\end{array}$ & No & $-80^{\circ} \mathrm{C}$ & No \\
\hline MARUYAMA [52] & 2012 & EDTA-ACD & $8000 \times g, 5 \mathrm{~min}$ & No & $-20^{\circ} \mathrm{C}$ & No \\
\hline
\end{tabular}

PPP: platelet-poor plasma; ACD: acid-citrate-dextrose. 
TABLE 2 Case-control trials investigating microparticle levels and phenotypes in obstructive sleep apnoea

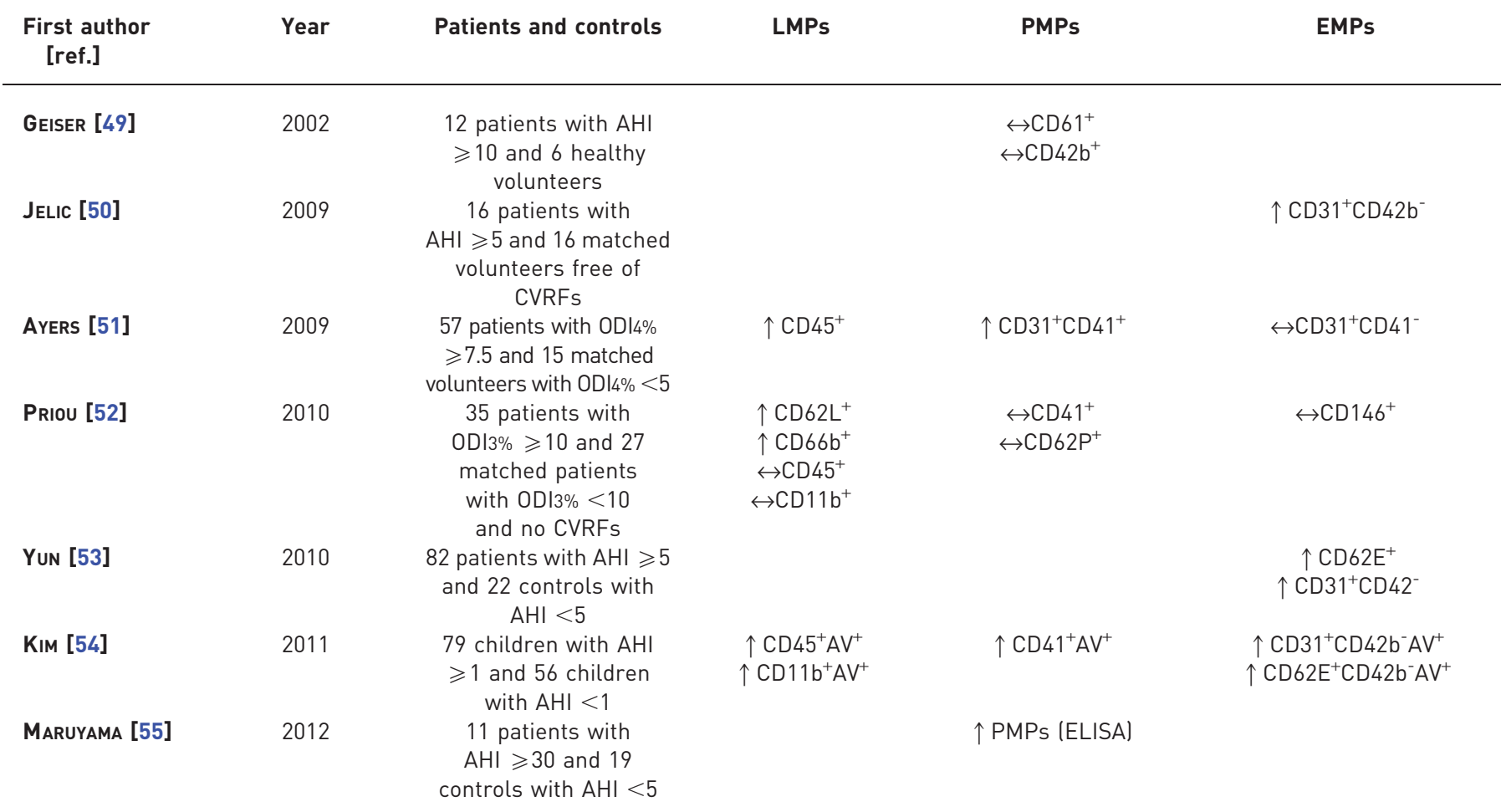

LMP: leukocyte-derived microparticle; PMP: platelet-derived microparticle; EMP: epithelium-derived microparticle; AHI: apnoea-hypopnoea index; CVRF: cardiovascular risk factor; ODIn\%: $n \%$ oxygen desaturation index; $\uparrow$ : increased; $\leftrightarrow$ : unchanged; AV: annexin V.

$[52,55]$ and four studies used citrate tubes $[49,50,51,53]$. The speed of centrifugation ranged from $1000 \times g$ [50] (MPs remain in the supernatant) to $20000 \times g$ [54] (MPs are pelleted) and the duration of the centrifugation ranged from 6 to $90 \mathrm{~min}$. Some protocols included further centrifugations for washing MPs, which could have contributed to MP loss [51]. None of the published data on MPs in OSA included information on previous centrifugation of the antibody solution. All studies showed no difference in the total number of MPs but marked differences were observed in their cellular origin.

\section{Platelet-derived MPs in OSA}

The characteristic surface markers that indicate a platelet origin are specific glycoproteins of platelets and megakaryocytes, such as CD41, CD62P (P-selectin), CD42b and CD61. Platelet-derived microparticles (PMPs) represent $60 \%$ of all MPs. Studies investigating PMP levels in OSA have reported conflicting results (table 2). In 2002, GEISER et al. [49] showed that although the percentage of platelets with the activationdependent epitope CD62P was increased during sleep (at 04:00 h) in OSA patients compared with controls, no significant difference was observed in $\mathrm{CD}_{2} \mathrm{~b}^{+}$or $\mathrm{CD}^{+} 1^{+} \mathrm{PMP}$ levels at either 04:00 $\mathrm{h}$ or 07:00 h. A recent case-control study by PRIOU et al. [52] also found no increase in $\mathrm{CD}_{4} 1^{+}$or $\mathrm{CD}_{2} \mathrm{P}^{+} \mathrm{PMP}_{\text {levels in }}$ OSA patients. In contrast, AYERS et al. [51], MARUYAMA et al. [55] and KIM et al. [54] found a significant increase in circulating $\mathrm{CD} 31^{+} \mathrm{CD} 41^{+}$and $\mathrm{CD} 41^{+} \mathrm{AV}^{+}$PMP levels in OSA patients. Furthermore, MARUYAMA et al. [55] and KIM et al. [54] found a positive correlation between PMP levels and OSA severity, as measured by the apnoea-hypopnea index (AHI). The effect of OSA treatment on PMPs is also controversial. MARUYAMA et al. [55] reported a decrease in PMP levels after 1 month of continuous positive airway pressure (CPAP) treatment in an uncontrolled study. In contrast, a randomised controlled study [57] showed no change in PMP levels after short-term CPAP withdrawal.

As discussed earlier, methodological differences could have contributed to the discordant results regarding PMP levels in OSA, as the centrifugation speeds were higher (the MPs were in the pellet) in studies demonstrating an increase in PMP levels in OSA patients, compared with the studies that investigated MPs in the supernatant. Discrepancies might also be due to the clinical characteristics of included patients. Indeed, PRIOU et al. [52] included patients and controls who were free from any cardiovascular disease or 
risk factors, whereas AYERS et al. [51] included patients with high body mass index (BMI), of whom 40\% were hypertensive and $10 \%$ had coronary heart disease. In the study by MARUYAMA et al. [55], the OSA and control subjects were not matched for BMI or cardiovascular risk factors. It can be hypothesised that metabolic comorbidities, also known to be associated with increased PMP levels [58], may have contributed to these conflicting results.

Little is known about the pathological relevance of changes in circulating PMP levels specifically in OSA. PMPs have been involved in the pathogenesis of inflammation and atherosclerosis [59] and to have a major role in blood coagulation [60,61]. PMP levels have been shown to be significantly associated with the vascular dysfunction linked to OSA in children [54] and could potentially account for the increased risk of altered endothelial function. In addition to their potential implication in the atherogenic process, PMPs could play a role in the increased blood coagulability described in OSA [62]. However, the clinical use of PMPs as reliable biomarker indicators of vascular risk will have to await further studies.

\section{Leukocyte-derived MPs}

Leukocyte-derived microparticles (LMPs) are derived from all leukocyte types, including neutrophils, monocytes, and B- and T-lymphocytes [63]. There are numerous characteristic surface markers that indicate a leukocyte origin, including: CD45 for pan-leukocyte-derived LMPs; CD66b for granulocytederived LMPs; CD11b for monocyte-, granulocyte-, macrophage- and natural killer cell-derived LMPs; and CD62L for activated leukocyte-derived LMPs.

LMP levels were increased in OSA patients compared with the controls in all published studies (table 2). In children with moderate-to-severe OSA, both $\mathrm{CD}_{11 b^{+}}$and $\mathrm{CD}_{4} 5^{+} \mathrm{LMP}$ levels were increased, and the CD $11 b^{+}$LMPs were positively correlated with OSA severity $(r=0.334, p<0.001)$ [54]. High levels of CD45 LMPs were also found in minimally symptomatic adults with OSA, compared with control subjects matched for the main anthropometric data [51]. In OSA patients with marked nocturnal desaturation, PRIOU et al. [52] described an increased level of granulocyte-derived LMPs expressing CD66b and activated leukocyte-derived LMPs expressing CD62L. Moreover, the CD62 $\mathrm{L}^{+} \mathrm{MP}$ levels were correlated with the oxygen desaturation index.

As increased LMP levels have been described in diseases such as diabetes and hypertension [64], these comorbid conditions may have contributed to the increased LMP levels in OSA. However, some investigators included OSA patients and controls who were closely matched for anthropomorphic data and cardiovascular risk factors [51] and/or free from any cardiovascular disease or risk factors [52]. TRZEPIZUR et al. [56] investigated the evening to morning changes in $\mathrm{CD}_{2} \mathrm{~L}^{+}$LMP levels. Whereas a decrease in $\mathrm{CD}_{62} \mathrm{~L}^{+}$LMP levels was observed in control subjects during the night, a significant increase was seen in patients with moderate-to-severe OSA, suggesting a nocturnal release of leukocyte-derived MPs in response to sleep disordered breathing. Furthermore, the authors found a significant correlation between the evening to morning change in $\mathrm{CD}_{2} \mathrm{~L}^{+} \mathrm{MP}$ levels and the $\mathrm{AHI}$ [56]. In a randomised controlled trial investigating the effect of 2 weeks of CPAP withdrawal in OSA patients, AYERS et al. [57] found a significant increase in granulocyte $\left(\mathrm{CD} 6 \mathrm{~b}^{+}\right)$LMP levels in the withdrawal group. Altogether, these findings provide strong evidence in support of a direct link between sleep disordered breathing and LMP levels.

As described more in detail later, in vitro incubation of endothelial cells with MPs from OSA patients was associated with a decrease in nitric oxide production that was correlated with circulating levels of CD62L $\mathrm{L}^{+}$ LMP [52]. In children with OSA, LMP levels correlated positively with vascular dysfunction [54]. Altogether, these findings suggest a specific role of LMPs in OSA-associated endothelial dysfunction.

\section{Endothelium-derived MPs}

The characteristic surface markers that indicate endothelial cell origin are numerous and disparate, and the majority are not specific for endothelial expression. Strategies that use a combination of multicolour antibodies have been proposed to overcome these difficulties. The various epitopes used are the presence of CD146 (S-endo), CD31 (platelet/endothelial cell adhesion molecule 1) and CD62E (E-selectin), associated with the absence of platelet characteristics (CD41 or CD42b).

Three groups have reported an increase in circulating levels of EMPs in OSA patients (table 2) [50, 53, 54]. JeLIC et al. [50] found that $\mathrm{CD} 31^{+} \mathrm{CD} 42 \mathrm{~b}^{-}$EMP levels were four-fold higher in patients with OSA compared with their matched controls and were negatively correlated with endothelial function, as assessed by flowmediated vasodilation. Furthermore, CPAP therapy tended to decrease CD $31^{+} \mathrm{CD} 42 \mathrm{~b}^{-}$EMP levels in patients with OSA compared with their baseline values. In the study by YUN et al. [53], CD $31^{+} \mathrm{CD}_{2} 2 \mathrm{~b}^{-}$and $\mathrm{CD}_{2} \mathrm{E}^{+}$EMP levels were significantly higher in OSA than in non-OSA patients. The AHI and the intimamedia thickness were positively correlated with $\mathrm{CD} 31^{+} \mathrm{CD} 41^{-}$EMP levels but not with $\mathrm{CD}_{2} \mathrm{E}^{+}$EMP levels. In 21 patients who consented to CPAP therapy, the authors observed a significant decrease in $\mathrm{CD}^{2} 2 \mathrm{E}^{+} \mathrm{EMP}$ 
levels but not in $\mathrm{CD}^{+} 1^{+} \mathrm{CD}_{4} \mathrm{~b}^{-}$EMP levels [53]. In the randomised controlled study by AYERS et al. [57], 2 weeks of CPAP withdrawal were associated with an increase in $\mathrm{CD}^{2} \mathrm{E}^{+}$EMP levels but not in $\mathrm{CD} 31^{+} \mathrm{CD} 41^{-}$EMP levels.

Two studies found no increase in EMP levels in OSA [52, 55]. The lack of increase in CD146 ${ }^{+}$EMP levels in the study from PRIOU et al. [52] may be explained by the fact that CD146, also known as the melanoma cell adhesion molecule, is a constitutive marker of the endothelial cell lineage and may be less adequate when investigating vascular damage than other markers such as CD31 and CD62E, which are associated with endothelial apoptosis and activation, respectively. The lack of increase in $\mathrm{CD} 31^{+} \mathrm{CD} 41^{-} \mathrm{EMPs}$ in the study by AYERS et al. [51] was observed in a selected population of minimally symptomatic OSA patients that may differ from sleepy OSA patients in terms of vascular and metabolic profile [65-67].

OSA is a complex disease, and various pathophysiological mechanisms are thought to contribute to the pathogenesis of the vascular impairment, including intermittent hypoxia, sympathetic overactivity, oxidative stress, systemic inflammation, hypertension, dyslipidaemia and insulin resistance. While hyperglycaemia and oxidative stress have been proposed to promote endothelial cell apoptosis [68], hypoxia and inflammation may induce endothelial cell activation [69]. Therefore, the impact of repeated apnoeas on endothelial cells may include both apoptosis and activation, and may be associated with the release of EMPs harbouring various corresponding epitopes. Some authors have suggested considering the ratio of $\mathrm{CD}_{2} \mathrm{E}^{+}$EMPs/CD31 $1^{+}$EMPs in order to differentiate between activating and apoptotic EMP profiles [43]. The application of this ratio in two previous studies investigating both $\mathrm{CD} 31^{+} \mathrm{CD} 42 \mathrm{~b}^{-} \mathrm{EMP}$ and CD62E+ EMP levels $[55,56]$ suggests an apoptotic EMP profile in children and adults with OSA. As markers of endothelial cell apoptosis, $\mathrm{CD} 31^{+} \mathrm{CD} 42 \mathrm{~b}^{-}$EMP levels are strongly correlated with OSA severity, endothelial dysfunction and carotid intima-media thickness [53, 54], and may reflect the chronic vascular damage induced by long-term exposure to repeated apnoeas. In contrast, CD62 $\mathrm{E}^{+} \mathrm{EMP}$ levels, reflecting endothelial cell activation, correlated neither with OSA nor with the severity of vascular damage but have been shown to be more sensitive to variation with CPAP initiation or withdrawal $[53,57]$. CD62E $\mathrm{E}^{+}$EMPs may reflect the acute impact of apnoeas on endothelial cell activation.

\section{MPs as biological vectors of vascular dysfunction in OSA Endothelial dysfunction}

There is good evidence, both in animal models of intermittent hypoxia and in humans, that OSA is associated with endothelial dysfunction, a pivotal element in the development and progression of atherosclerosis [70-72]. Experimental data have demonstrated that MPs can promote endothelial dysfunction. MPs released in vitro by apoptotic T-lymphocytes impair endothelial function by stimulating oxygen free radical generation and decreasing Ser 1179 phosphorylation of endothelial nitric oxide synthase (eNOS) [73, 74]. By collecting blood samples from patients suspected of OSA, PRIOU et al. [52] showed that MPs from OSA patients with marked nocturnal desaturation induced ex vivo endothelial dysfunction in the aorta and small mesenteric arteries when injected into mice. In vitro, incubation of endothelial cells with MPs from OSA patients for $24 \mathrm{~h}$ caused decreased nitric oxide production, independently of oxidative stress. Furthermore, the decrease in cellular nitric oxide production correlated with the CD62L $\mathrm{L}^{+} \mathrm{LMP}$ circulating levels, suggesting a specific role of CD62L $\mathrm{L}^{+}$LMPs in OSA-associated endothelial dysfunction [52].

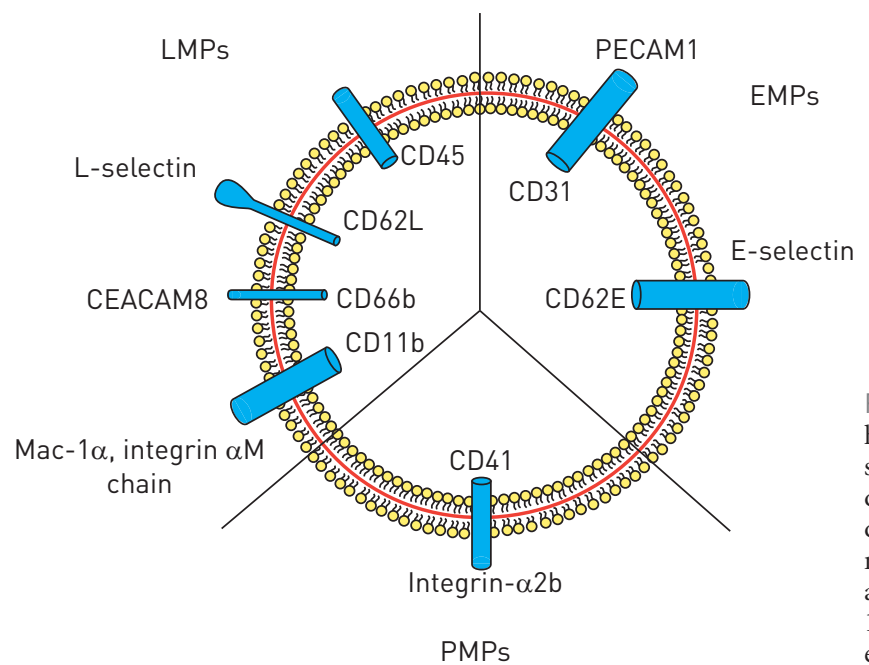

FIGURE 1 Illustration of the main epitopes harboured by microparticles from obstructive sleep apnoea patients. LMP: leukocytederived microparticle; EMP: endotheliumderived microparticle; PMP: platelet-derived microparticle; CEACAM: carcinoembryonic antigen-related cell adhesion molecule; Mac1: macrophage antigen-1; PECAM: platelet endothelial cell adhesion molecule. 


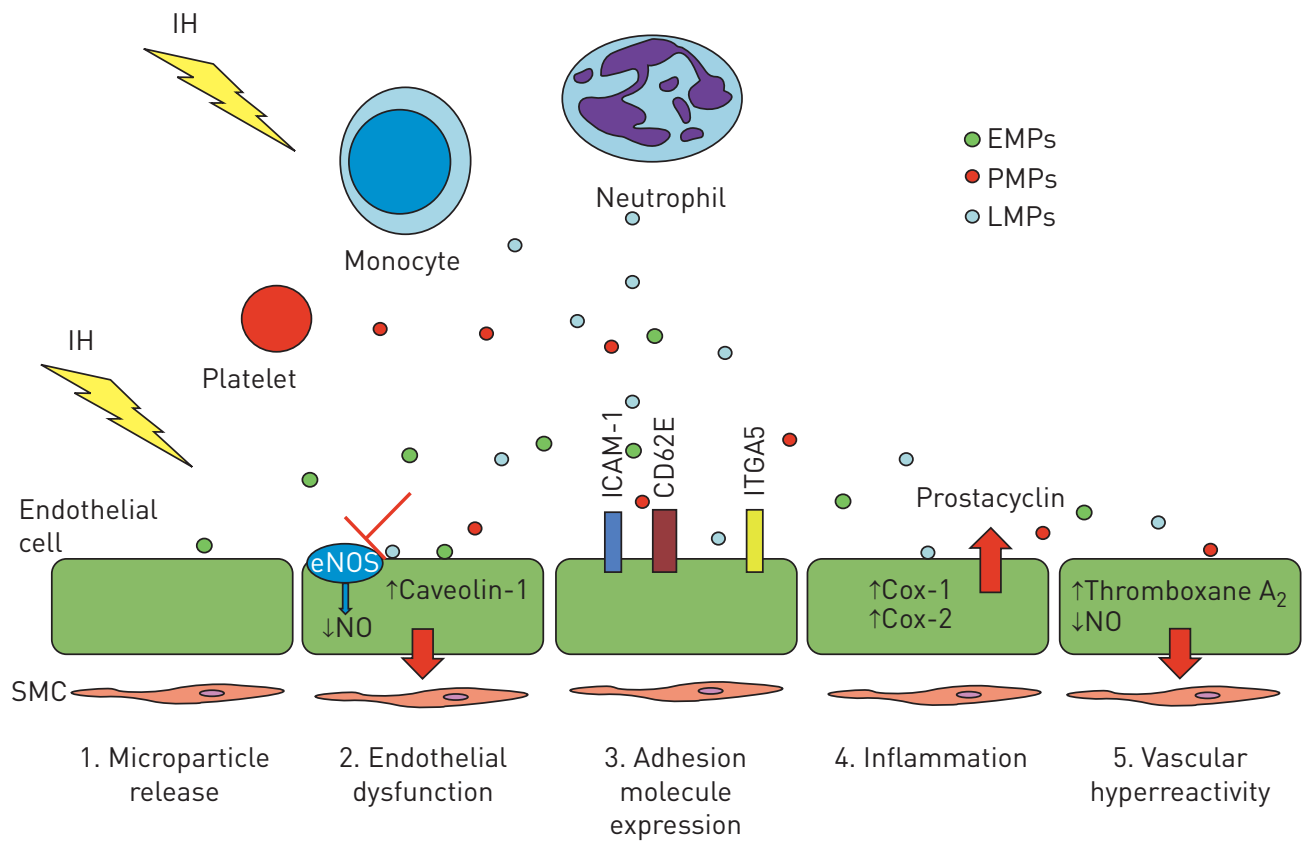

FIGURE 2 Illustration of the main pathways through which circulating microparticles (MPs) may contribute to the obstructive sleep apnoea (OSA)-associated vascular dysfunction. 1) OSAs induce intermittent hypoxia (IH), which may stimulate the release of platelet-derived microparticles (PMPs), endothelium-derived microparticles (EMPs) and leukocyte-derived microparticles (LMPs). 2) Circulating MPs interact with endothelial cells and contribute to impair endothelial function by enhancing phosphorylation of endothelial nitric oxide synthase (eNOS) at the site of inhibition and inducing expression of caveolin-1.3) MPs promote the cell-cell interactions by increasing expression of the vascular adhesive molecules: intercellular adhesion molecule (ICAM)-1, CD62E (E-selectin) and integrin- $\alpha 5$ (ITGA5). 4) MPs promote vascular inflammation by increasing cyclooxygenase (Cox) expression and prostacyclin release. 5) MPs lead to vascular hyperreactivity by reducing endothelial nitric oxide (NO) release and promoting the secretion of vasoconstrictors. SMC: smooth muscle cell.

In a subsequent study [75], MPs from OSA patients injected into mice induced ex vivo vascular hyperreactivity in aortas with a functional endothelium but not in vessels without a functional endothelium, highlighting the mandatory role of the endothelium. Molecular investigations demonstrated that MPs from OSA patients reduced eNOS activity and subsequent nitric oxide production, increased aortic cyclooxygenase (Cox)-1 and Cox-2 expression, and increased thromboxane $\mathrm{A}_{2}$ and prostacyclin production. Altogether, these findings support a potential implication of circulating MPs in OSA-associated endothelial dysfunction.

\section{Vascular inflammation}

There is evidence that OSA is associated with vascular inflammation, which is thought to be a major component of the atherogenic process. OSA patients have elevated serum levels of TNF- $\alpha$, interleukin (IL)-6, IL-8, C-reactive protein [76, 77] and adhesion molecules (CD62L, soluble CD62E, CD62P, intercellular adhesion molecule (ICAM)-1 and vascular cell adhesion molecule (VCAM)-1) [77, 78]. OSA is also associated with delayed neutrophil apoptosis and increased levels of adhesion molecules, which could contribute to enhanced neutrophil-leukocyte interactions, and facilitate free radical production and proteolytic release [79].

MPs are key factors in the inflammatory process, and contribute to the endothelial production of various pro-inflammatory cytokines and chemokines. MPs isolated from human atherosclerotic plaques were found to stimulate the in vitro release of IL-1 $\beta$, IL- 6 and CCL2, as well as inducing the expression of ICAM-1, VCAM-1 and CD62E [16, 80]. When incubated for $24 \mathrm{~h}$ with endothelial cells, MPs isolated from OSA patients induced overexpression of pro-inflammatory molecules, including CD62E, Cox-2 and ICAM-1 [52]. When injected into mice, MPs from OSA patients induced overexpression of pro-inflammatory enzymes, such as Cox-1 and Cox- 2 in the aorta, and overproduction of pro-inflammatory cytokines in the supernatants from the animal's aorta (thromboxane $\mathrm{A}_{2}, 8$-isoprostane, prostacyclin and prostaglandin $\mathrm{E}_{2}$ ) [75]. Altogether, these findings support a role for MPs in the vascular inflammation associated with OSA. 


\section{Oxidative stress}

It has been clearly established that atherogenesis is linked to oxidative stress and lipid peroxidation [81]. In animal models of OSA, intermittent hypoxia increased reactive oxygen species (ROS) generation in the vascular wall [82], induced lipid peroxidation [83] and induced the formation of oxidised low-density lipoprotein (LDL)-cholesterol, creating a substrate for atherosclerotic plaques [84]. JELIC et al. [85] showed increased oxidative stress in endothelial cells harvested from the vessels of OSA patients. Increased lipid peroxidation and elevated levels of oxidised LDL were observed in OSA patients [86].

MPs are able to modulate oxidative stress. Incubation of endothelial cells with LMPs from apoptotic T-lymphocytes enhanced ROS production through a xanthine oxidase-sensitive mechanism [74, 87]. In contrast, in vitro treatment of endothelial cells with MPs from patients with the metabolic syndrome reduced both nitric oxide and superoxide anion production, resulting in protein tyrosine nitration [87]. However, PRIOU et al. [52] studied in vitro superoxide anion production by endothelial cells incubated with MPs from OSA patients and controls, and found no significant differences.

\section{Conclusion and perspectives}

Clinical studies have shown that levels of MPs of various cellular origins, including platelets, endothelial cells and leukocytes, are increased in OSA patients. Figure 1 illustrates the main antigenic epitopes harboured by MPs investigated in OSA patients. Levels of MPs harbouring markers of cellular activation or apoptosis that are known to predict poor cardiovascular outcomes were found to be correlated with OSA severity as well as markers of vascular impairment, and were modified by OSA treatment. As potential biomarkers of vascular dysfunction, MPs could provide a useful tool to predict cardiovascular outcome and monitor treatment response in OSA patients. However, the clinical relevance of MP measurement is currently hampered by methodological concerns. Major discrepancies between studies investigating MPs in OSA patients may be due to the wide heterogeneity in pre-analytical and analytical processes, which need to be standardised.

Experimental data suggest that MPs may contribute to the pathophysiology of OSA-associated vascular impairment by promoting endothelial dysfunction, inflammation and vascular hyperreactivity (figure 2). Thus, MPs may emerge as a novel biological vector of vascular dysfunction adding another layer of complexity to the already multifaceted mechanisms involved in OSA-associated vascular morbidity. Further studies are required to investigate the pathways through which MPs may impair vascular function in OSA and to determine whether MPs could constitute a novel therapeutic target to improve cardiovascular outcome in OSA.

\section{References}

1 Marin JM, Carrizo SJ, Vicente E, et al. Long-term cardiovascular outcomes in men with obstructive sleep apnoeahypopnoea with or without treatment with continuous positive airway pressure: an observational study. Lancet 2005; 365: 1046-1053.

2 Marshall NS, Wong KK, Liu PY, et al. Sleep apnea as an independent risk factor for all-cause mortality: the Busselton Health Study. Sleep 2008; 31: 1079-1085.

3 Punjabi NM, Caffo BS, Goodwin JL, et al. Sleep-disordered breathing and mortality: a prospective cohort study. PLoS Med 2009; 6: e1000132.

4 Gottlieb DJ, Yenokyan G, Newman AB, et al. Prospective study of obstructive sleep apnea and incident coronary heart disease and heart failure: the Sleep Heart Health Study. Circulation 2010; 122: 352-360.

5 Redline S, Yenokyan G, Gottlieb DJ, et al. Obstructive sleep apnea-hypopnea and incident stroke: the Sleep Heart Health Study. Am J Respir Crit Care Med 2010; 182: 269-277.

6 Chami HA, Devereux RB, Gottdiener JS, et al. Left ventricular morphology and systolic function in sleepdisordered breathing: the Sleep Heart Health Study. Circulation 2008; 117: 2599-2607.

7 Wachter R, Luthje L, Klemmstein D, et al. Impact of obstructive sleep apnoea on diastolic function. Eur Respir J 2013; 41: 376-383.

8 Rossi VA, Stradling JR, Kohler M. Effects of obstructive sleep apnoea on heart rhythm. Eur Respir J 2013; 41: 1439-1451.

9 Savransky V, Nanayakkara A, Li J, et al. Chronic intermittent hypoxia induces atherosclerosis. Am J Respir Crit Care Med 2007; 175: 1290-1297.

10 Jun J, Reinke C, Bedja D, et al. Effect of intermittent hypoxia on atherosclerosis in apolipoprotein E-deficient mice. Atherosclerosis 2010; 209: 381-386.

11 Li J, Savransky V, Nanayakkara A, et al. Hyperlipidemia and lipid peroxidation are dependent on the severity of chronic intermittent hypoxia. J Appl Physiol 2007; 102: 557-563.

12 Trzepizur W, Le Vaillant M, Meslier N, et al. Independent association between nocturnal intermittent hypoxemia and metabolic dyslipidemia. Chest 2013; 143: 1584-1589.

13 Iiyori $\mathrm{N}$, Alonso LC, Li J, et al. Intermittent hypoxia causes insulin resistance in lean mice independent of autonomic activity. Am J Respir Crit Care Med 2007; 175: 851-857.

14 Dematteis M, Julien C, Guillermet C, et al. Intermittent hypoxia induces early functional cardiovascular remodeling in mice. Am J Respir Crit Care Med 2008; 177: 227-235.

15 Camaioni C, Gustapane M, Cialdella P, et al. Microparticles and microRNAs: new players in the complex field of coagulation. Intern Emerg Med 2013; 8: 291-296. 
Rautou PE, Leroyer AS, Ramkhelawon B, et al. Microparticles from human atherosclerotic plaques promote endothelial ICAM-1-dependent monocyte adhesion and transendothelial migration. Circ Res 2011; 108: 335-343.

17 Faille D, El-Assaad F, Mitchell AJ, et al. Endocytosis and intracellular processing of platelet microparticles by brain endothelial cells. J Cell Mol Med 2012; 16: 1731-1738.

18 Quesenberry PJ, Aliotta JM. Cellular phenotype switching and microvesicles. Adv Drug Deliv Rev 2010; 62: 1141-1148.

19 Tual-Chalot S, Leonetti D, Andriantsitohaina R, et al. Microvesicles: intercellular vectors of biological messages. Mol Interv 2011; 11: 88-94.

20 Martinez MC, Tual-Chalot S, Leonetti D, et al. Microparticles: targets and tools in cardiovascular disease. Trends Pharmacol Sci 2011; 32: 659-665.

21 Martinez MC, Andriantsitohaina R. Microparticles in angiogenesis: therapeutic potential. Circ Res 2011; 109: 110-119.

22 Andriantsitohaina R, Gaceb A, Vergori L, et al. Microparticles as regulators of cardiovascular inflammation. Trends Cardiovasc Med 2012; 22: 88-92.

23 Owens AP 3rd, Mackman N. Microparticles in hemostasis and thrombosis. Circ Res 2011; 108: $1284-1297$.

24 Augustine D, Ayers LV, Lima E, et al. Dynamic Release and Clearance of Circulating Microparticles During Cardiac Stress. Circ Res 2014; 114: 109-113.

25 Mostefai HA, Meziani F, Mastronardi ML, et al. Circulating microparticles from patients with septic shock exert protective role in vascular function. Am J Respir Crit Care Med 2008; 178: 1148-1155.

26 Perez-Casal M, Thompson V, Downey C, et al. The clinical and functional relevance of microparticles induced by activated protein C treatment in sepsis. Crit Care 2011; 15: R195.

27 Amabile N, Boulanger CM. Circulating microparticle levels in patients with coronary artery disease: a new indicator of vulnerability? Eur Heart J 2011; 32: 1958-1960.

28 Morel O, Pereira B, Averous G, et al. Increased levels of procoagulant tissue factor-bearing microparticles within the occluded coronary artery of patients with ST-segment elevation myocardial infarction: role of endothelial damage and leukocyte activation. Atherosclerosis 2009; 204: 636-641.

29 Boulanger CM. Microparticles, vascular function and hypertension. Curr Opin Nephrol Hypertens 2010; 19: 177-180.

30 Nomura S. Dynamic role of microparticles in type 2 diabetes mellitus. Curr Diabetes Rev 2009; 5: 245-251.

31 McVey M, Tabuchi A, Kuebler WM. Microparticles and acute lung injury. Am J Physiol Lung Cell Mol Physiol 2012; 303: L364-L381.

32 Amabile N, Guignabert C, Montani D, et al. Cellular microparticles in the pathogenesis of pulmonary hypertension. Eur Respir J 2013; 42: 272-279.

33 Chironi G, Simon A, Hugel B, et al. Circulating leukocyte-derived microparticles predict subclinical atherosclerosis burden in asymptomatic subjects. Arterioscler Thromb Vasc Biol 2006; 26: 2775-2780.

34 Lee ST, Chu K, Jung KH, et al. Circulating CD62E ${ }^{+}$microparticles and cardiovascular outcomes. PLoS One 2012; 7: e35713.

35 Ferreira AC, Peter AA, Mendez AJ, et al. Postprandial hypertriglyceridemia increases circulating levels of endothelial cell microparticles. Circulation 2004; 110: 3599-3603.

36 Chaar V, Romana M, Tripette J, et al. Effect of strenuous physical exercise on circulating cell-derived microparticles. Clin Hemorheol Microcirc 2011; 47: 15-25.

37 Ayers L, Kohler M, Harrison P, et al. Measurement of circulating cell-derived microparticles by flow cytometry: sources of variability within the assay. Thromb Res 2011; 127: 370-377.

38 Dey-Hazra E, Hertel B, Kirsch T, et al. Detection of circulating microparticles by flow cytometry: influence of centrifugation, filtration of buffer, and freezing. Vasc Health Risk Manag 2010; 6: 1125-1133.

39 Lacroix R, Judicone C, Poncelet P, et al. Impact of pre-analytical parameters on the measurement of circulating microparticles: towards standardization of protocol. J Thromb Haemost 2012; 10: 437-446.

40 Jayachandran M, Miller VM, Heit JA, et al. Methodology for isolation, identification and characterization of microvesicles in peripheral blood. J Immunol Methods 2012; 375: 207-214.

41 Stagnara J, Garnache Ottou F, Angelot F, et al. Correlation between platelet-derived microparticle enumeration by flow cytometry and phospholipid-dependent procoagulant activity in microparticles: the centrifugation step matters! Thromb Haemost 2012; 107: 1185-1187.

42 Lacroix R, Judicone C, Mooberry M, et al. Standardization of pre-analytical variables in plasma microparticle determination: results of the International Society on Thrombosis and Haemostasis SSC Collaborative workshop. J Thromb Haemost 2013; 11: 1190-1193.

43 Jimenez JJ, Jy W, Mauro LM, et al. Endothelial cells release phenotypically and quantitatively distinct microparticles in activation and apoptosis. Thromb Res 2003; 109: 175-180.

44 Bernal-Mizrachi L, Jy W, Jimenez JJ, et al. High levels of circulating endothelial microparticles in patients with acute coronary syndromes. Am Heart J 2003; 145: 962-970.

45 Horstman LL, Jy W, Jimenez JJ, et al. New horizons in the analysis of circulating cell-derived microparticles. Keio J Med 2004; 53: 210-230.

46 Wang W, Singh S, Zeng DL, et al. Antibody structure, instability, and formulation. J Pharm Sci $2007 ; 96$ : 1-26.

47 Aass HC, Ovstebo R, Troseid AM, et al. Fluorescent particles in the antibody solution result in false TF- and CD14positive microparticles in flow cytometric analysis. Cytometry A 2011; 79: 990-999.

48 Lacroix R, Robert S, Poncelet P, et al. Overcoming limitations of microparticle measurement by flow cytometry. Semin Thromb Hemost 2012; 36: 807-818.

49 Geiser T, Buck F, Meyer BJ, et al. In vivo platelet activation is increased during sleep in patients with obstructive sleep apnea syndrome. Respiration 2002; 69: 229-234.

50 Jelic S, Lederer DJ, Adams T, et al. Endothelial repair capacity and apoptosis are inversely related in obstructive sleep apnea. Vasc Health Risk Manag 2009; 5: 909-920.

51 Ayers L, Ferry B, Craig S, et al. Circulating cell-derived microparticles in patients with minimally symptomatic obstructive sleep apnoea. Eur Respir J 2009; 33: 574-580.

52 Priou P, Gagnadoux F, Tesse A, et al. Endothelial dysfunction and circulating microparticles from patients with obstructive sleep apnea. Am J Pathol 2010; 177: 974-983.

53 Yun $\mathrm{CH}$, Jung $\mathrm{KH}$, Chu K, et al. Increased circulating endothelial microparticles and carotid atherosclerosis in obstructive sleep apnea. J Clin Neurol 2010; 6: 89-98. 
breathing. Chest 2011; 140: 408-417.

55 Maruyama K, Morishita E, Sekiya A, et al. Plasma levels of platelet-derived microparticles in patients with obstructive sleep apnea syndrome. J Atheroscler Thromb 2012; 19: 98-104.

56 Trzepizur W, Priou P, Paris A, et al. Nocturnal release of leukocyte-derived microparticles in males with obstructive sleep apnoea. Eur Respir J 2011; 37: 1293-1295.

57 Ayers L, Stoewhas AC, Ferry B, et al. Elevated levels of endothelial cell-derived microparticles following short-term withdrawal of continuous positive airway pressure in patients with obstructive sleep apnea: data from a randomized controlled trial. Respiration 2013; 85: 478-485.

Agouni A, Ducluzeau PH, Benameur T, et al. Microparticles from patients with metabolic syndrome induce vascular hypo-reactivity via Fas/Fas-ligand pathway in mice. PLoS One 2011; 6: e27809.

59 Nomura S, Tandon NN, Nakamura T, et al. High-shear-stress-induced activation of platelets and microparticles enhances expression of cell adhesion molecules in THP-1 and endothelial cells. Atherosclerosis 2001; 158: $277-287$.

60 Lee YJ, Jy W, Horstman LL, et al. Elevated platelet microparticles in transient ischemic attacks, lacunar infarcts, and multiinfarct dementias. Thromb Res 1993; 72: 295-304.

61 Geiser T, Sturzenegger M, Genewein U, et al. Mechanisms of cerebrovascular events as assessed by procoagulant activity, cerebral microemboli, and platelet microparticles in patients with prosthetic heart valves. Stroke 1998; 29: 1770-1777.

62 Liak C, Fitzpatrick M. Coagulability in obstructive sleep apnea. Can Respir J 2011; 18: 338-348.

63 Angelillo-Scherrer A. Leukocyte-derived microparticles in vascular homeostasis. Circ Res 2012; 110: 356-369.

64 Nomura S, Kanazawa S, Fukuhara S. Effects of efonidipine on platelet and monocyte activation markers in hypertensive patients with and without type 2 diabetes mellitus. J Hum Hypertens 2002; 16: 539-547.

65 Montserrat JM, Garcia-Rio F, Barbe F. Diagnostic and therapeutic approach to nonsleepy apnea. Am J Respir Crit Care Med 2007; 176: 6-9.

66 Barbe F, Mayoralas LR, Duran J, et al. Treatment with continuous positive airway pressure is not effective in patients with sleep apnea but no daytime sleepiness. a randomized, controlled trial. Ann Intern Med 2001; 134: 1015-1023.

67 Robinson GV, Smith DM, Langford BA, et al. Continuous positive airway pressure does not reduce blood pressure in nonsleepy hypertensive OSA patients. Eur Respir J 2006; 27: 1229-1235.

68 Hogg N, Browning J, Howard T, et al. Apoptosis in vascular endothelial cells caused by serum deprivation, oxidative stress and transforming growth factor- $\beta$. Endothelium 1999; 7: 35-49.

69 Ryan S, Taylor CT, McNicholas WT. Selective activation of inflammatory pathways by intermittent hypoxia in obstructive sleep apnea syndrome. Circulation 2005; 112: 2660-2667.

70 Kohler M, Stoewhas AC, Ayers L, et al. Effects of continuous positive airway pressure therapy withdrawal in patients with obstructive sleep apnea: a randomized controlled trial. Am J Respir Crit Care Med 2011; 184: 1192-1199.

71 Cross MD, Mills NL, Al-Abri M, et al. Continuous positive airway pressure improves vascular function in obstructive sleep apnoea/hypopnoea syndrome: a randomised controlled trial. Thorax 2008; 63: 578-583.

72 Drager LF, Polotsky VY, Lorenzi-Filho G. Obstructive sleep apnea: an emerging risk factor for atherosclerosis. Chest 2011; 140: 534-542.

73 Martin S, Tesse A, Hugel B, et al. Shed membrane particles from T lymphocytes impair endothelial function and regulate endothelial protein expression. Circulation 2004; 109: 1653-1659.

74 Mostefai HA, Agouni A, Carusio N, et al. Phosphatidylinositol 3-kinase and xanthine oxidase regulate nitric oxide and reactive oxygen species productions by apoptotic lymphocyte microparticles in endothelial cells. J Immunol 2008; 180: 5028-5035.

75 Tual-Chalot S, Fatoumata K, Priou P, et al. Circulating microparticles from patients with obstructive sleep apnea enhance vascular contraction: mandatory role of the endothelium. Am J Pathol 2012; 181: 1473-1482.

76 Shamsuzzaman AS, Winnicki M, Lanfranchi P, et al. Elevated C-reactive protein in patients with obstructive sleep apnea. Circulation 2002; 105: 2462-2464.

77 Drager LF, Lopes HF, Maki-Nunes C, et al. The impact of obstructive sleep apnea on metabolic and inflammatory markers in consecutive patients with metabolic syndrome. PLoS One 2010; 5: e12065.

78 Ohga E, Tomita T, Wada H, et al. Effects of obstructive sleep apnea on circulating ICAM-1, IL-8, and MCP-1. J Appl Physiol 2003; 94: 179-184.

79 Dyugovskaya L, Polyakov A, Lavie P, et al. Delayed neutrophil apoptosis in patients with sleep apnea. Am J Respir Crit Care Med 2008; 177: 544-554.

80 MacKenzie A, Wilson HL, Kiss-Toth E, et al. Rapid secretion of interleukin-1 $\beta$ by microvesicle shedding. Immunity 2001; 15: 825-835.

81 Stocker R, Keaney JF Jr. Role of oxidative modifications in atherosclerosis. Physiol Rev 2004; 84: 1381-1478.

82 Savransky V, Jun J, Li J, et al. Dyslipidemia and atherosclerosis induced by chronic intermittent hypoxia are attenuated by deficiency of stearoyl coenzyme A desaturase. Circ Res 2008; 103: 1173-1180.

83 Jun J, Savransky V, Nanayakkara A, et al. Intermittent hypoxia has organ-specific effects on oxidative stress. Am J Physiol Regul Integr Comp Physiol 2008; 295: R1274-R1281.

84 Tan KC, Chow WS, Lam JC, et al. HDL dysfunction in obstructive sleep apnea. Atherosclerosis 2006; 184: 377-382.

85 Jelic S, Padeletti M, Kawut SM, et al. Inflammation, oxidative stress, and repair capacity of the vascular endothelium in obstructive sleep apnea. Circulation 2008; 117: 2270-2278.

86 Lavie L, Vishnevsky A, Lavie P. Evidence for lipid peroxidation in obstructive sleep apnea. Sleep 2004; 27 : 123-128.

87 Agouni A, Lagrue-Lak-Hal AH, Ducluzeau PH, et al. Endothelial dysfunction caused by circulating microparticles from patients with metabolic syndrome. Am J Pathol 2008; 173: 1210-1219. 